\title{
Facile nitrogen-free one-potaqueous synthesis of highly soluble and luminescent CdSe quantum dots
}

\author{
Elhussein M. Hashem*, Mohamed A. Ahmed and Meshel F. Abdel Messih
}

Chemistry Department, Faculty of Science, Ain-Shams University, Cairo, Egypt

*Corresponding Author E-mail: elhusseinhashem_p@sci.asu.edu.eg

Received: April 29, 2020

Accepted: June 16, 2020

CdSe quantum dots (QDs) have been widely used in a plethora of applications due to their relatively high photocatalytic abilities and unique properties. Herein, we report that highly water-soluble CdSe (QDs) were successfully prepared in an open-air condition without using an inert atmosphere. This has been accomplished through a facile one-pot method by using a novel binary mixture of thioglycolic acid (TGA) and trisodium citrate (TSC) as capping agents. Air stable and cheap sodium selenite $\left(\mathrm{Na}_{2} \mathrm{SeO}_{3}\right)$ was used as the selenium precursor. Structural and morphological properties of the as-prepared QDs were examined by varying the reflux time and the temporal evolution of the optical. The obtained QDs were characterized by XRD, HRTEM, XPS, UV-Vis absorption, DRS and PL. The results revealed that we can use this facile 'green' approach in synthesis high quality, small, spherical and monodispersed QDs which exhibits high optical properties and superior stability in open-air conditions.

Keywords: Quantum dots, CdSe, Semiconductors, Photocayalysis.

\section{INTRODUCTION}

Green and efficient energy technologies are a crucial point where nanoscience could make a difference in the paradigm shift from the traditional strategies to the advance oxidations processes (AOPs) ${ }^{[1,2]}$. Moreover, narrow bandgap semiconductors QDs are of great interest for numerous research and technical applications due to their quantum size effect, size-tunable PL spectrum, narrow PL bands and broad absorption spectrum in the visible region ${ }^{[3,4]}$. Amongst them, CdSe colloidal QDs have been studied the most due to their emission which can be easily tuned from blue to red range according to its size and alternatively its significant low bandgap energy (1.74 in bulk phase). This has led to attracting attention to its usage as a promising candidate in fundamental studies and applications such as solar cells, hydrogen production, biological imaging, wastewater treatment, LED and laser applications ${ }^{[5-9]}$. Although high-quality CdSe QDs which possess monodisperse sizes, shapes, high intensity and narrow PL peaks were reproducibly and controllably prepared by using organometallic route ${ }^{[10]}$ or its alternative approach ${ }^{[11]}$, the reports regarding the synthesis of CdSe QDs using the above schemes are exhibiting major problems and demerits such as utilization of high temperature needed to the crystal growth or using hazardous capping agents such as TOPO $\mathrm{TBP}^{[17,18]}$ and environmental unfriendly solvents such as Oleic acid, Toluene, n-Hexane as solvents excluding its employing in biological applications ${ }^{[14-16]}$.

Recently, thiols were utilized as promising capping agents in the water-based synthesis of QDs owing to their non-toxicity, and their high tendency to bind to their surface ${ }^{[17,18]}$.Moreover, the nucleation formation rapidly occurs at room temperature and the crystals slowly grow at low temperatures $\left(\sim 100^{\circ} \mathrm{C}\right)^{[19]}$. So, it is reasonable to choose the green aqueous route as an applicable method to controllably synthesize QDs at large scale. Due to 
Elhussein M. Hashem et al.

good water solubility and biocompatibility trisodium citrate ${ }^{[20]}$, and different thiols have been utilized in CdSe QDs preparation such as thioglycolic acid (TGA) ${ }^{[19]}$, 2-Mercabtoethanol ${ }^{[21]}$, 3 -Mercaptopropionic acid ${ }^{[22]}$, bio-thiols like cysteine ${ }^{[23]}$, and Glutathione ${ }^{[24]}$.

Herein, in this study, green and simple strategy were used for synthesis of highly water-soluble and luminescent CdSe QDs using a novel binary mixture of TGA and trisodium citrate, besides the employing of sodium selenite as a cheap Se precursor instead of the expensive sources like Se metal and the insoluble $\mathrm{SeO}$. The synthesis was carried out in open-air conditions, low temperature during reflux $\left(90^{\circ} \mathrm{C}\right)$ and showed excellent stability against oxidation.

\section{MATERIALS AND METHODS}

\section{Materials}

Both cadmium acetate dihydrate $(\mathrm{Cd}(\mathrm{CH} 3 \mathrm{COO}) 2.2 \mathrm{H} 2 \mathrm{O})$ and trisodium citrate 5.5hydrate (TSC, C6H5Na3O7.5.5H2O) were purchased from Merck. Sodium selenite (Na2SeO3) was purchased from ProLabo, and Thioglycolic acid (HSCH2COOH) (TGA) was purchased from PanreacQuimica. Sodium boron hydride (NaBH4) and sodium hydroxide $(\mathrm{NaOH})$ were of pure grade and used without any further purification.

\section{Synthesis of CdSe QDs}

A facile green nitrogen-free one-pot route was utilized to synthesize highly soluble and luminescent CdSe QDs. Firstly, $3 \mathrm{mmol}$ of cadmium acetate, $27.6 \mathrm{mmol}$ TGA, and $9 \mathrm{mmol}$ of TSC were dissolved in $200 \mathrm{~mL}$ of deionized water, then the mixture was magnetically stirred for $1 \mathrm{~h}$. The $\mathrm{pH}$ was adjusted to 12 by $1 \mathrm{M} \mathrm{NaOH}$ solution. Secondly, the selenium precursor was prepared by mixing $1 \mathrm{mmol}$ of sodium selenite $(\mathrm{Na} 2 \mathrm{SeO} 3)$ and $0.5 \mathrm{~g}$ of sodium boron hydride (NaBH4) in $200 \mathrm{~mL}$ deionized water, the solution was magnetically stirred till the red color appear. After that, the freshly prepared selenium precursor solution was slowly added to the previous mixture. The desired yellow solution of QDs was obtained instantaneously, and by refluxing the mixture for $4 \mathrm{~h}$ at $90^{\circ} \mathrm{C}$, the color of solution varies from yellow to orange to red over the time, at each hour a sample was taken for comparison; 5 samples were withdrawn and labeled $(0 \mathrm{~h}, 1 \mathrm{~h}, 2 \mathrm{~h}, 3 \mathrm{~h}, 4 \mathrm{~h})$ according to the time of reflux of each sample. The desired QDs at each time were separated by adding an equal amount of methanol. The powder was collected by centrifuge, further washed several times with methanol then dried at room temperature.

\section{XRD}

\section{RESULTS AND DISCUSSIONS}

Figure (1 a). displays the XRD patterns of the as-prepared CdSe QDs. XRD shows 3 main peaks at $2 \theta=25.4^{\circ}, 42^{\circ}, 49.7^{\circ}$ which are corresponding to the (111), (220), and (311) planes of the cubic CdSe (JCPDS No. 19-0191). Notably, the crystallinity of the samples increased with increasing the refluxing time; the main peaks are becoming sharper through the recrystallization process. The average crystallite sizes of the synthesized QDs calculated from the (111) peak of XRD using the Debye-Scherrer equation is about 2.2, 5.2, 5.7, 7.8, and $9 \mathrm{~nm}$, respectively. 

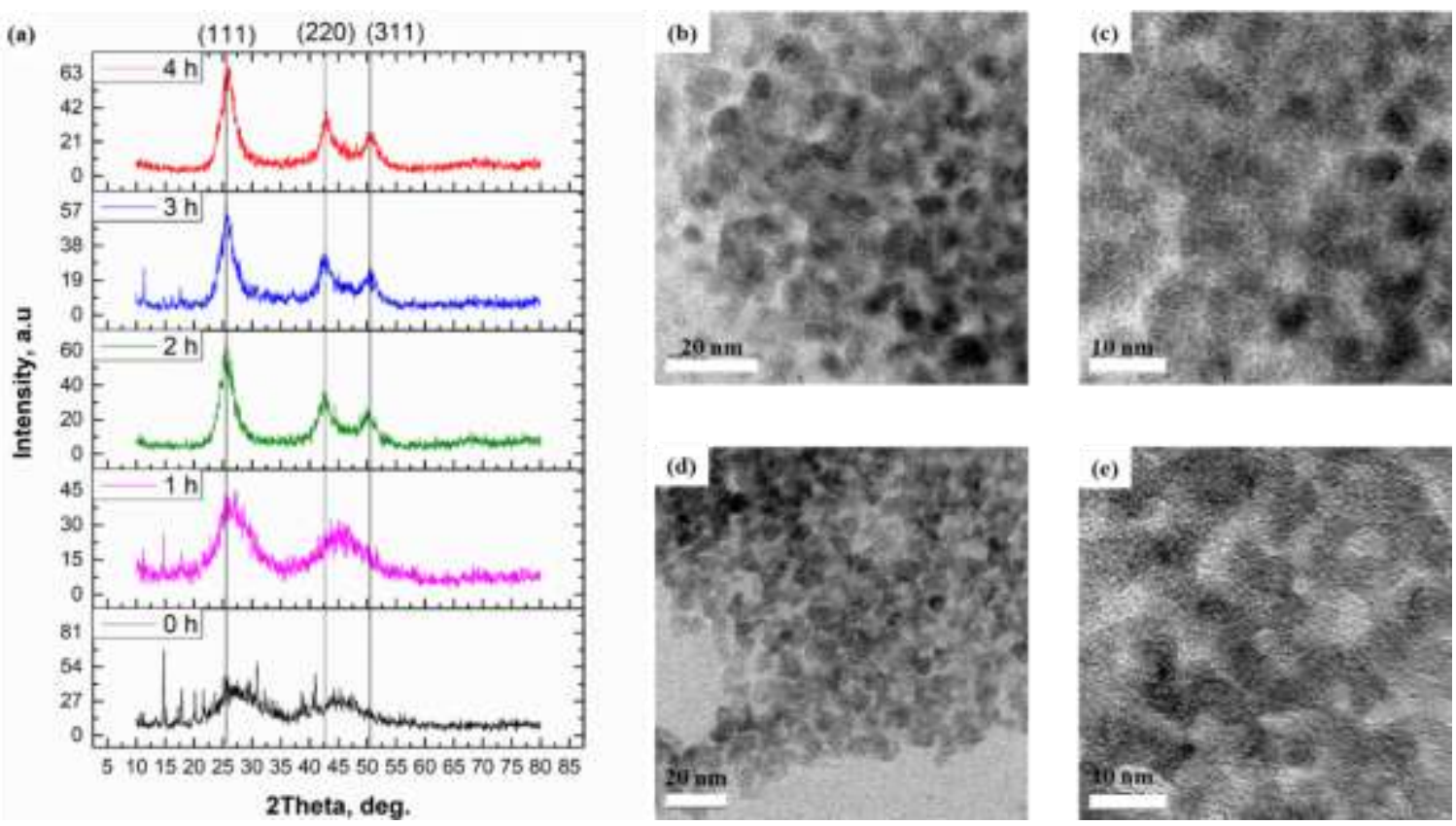

Fig. 1. The XRD patterns of the as-prepared CdSe QDs 0-4 h (a), and the corresponding HR-TEM images of $0 \mathrm{~h}(\mathrm{~b}-\mathrm{c})$ and $4 \mathrm{~h}$ (d-e) samples.

\section{HR-TEM}

Nevertheless, the morphology of the as-prepared CdSeQDs was demonstrated in the HRTEM images (Fig. 1 b-e). These images reflect the small crystallites, well-dispersed and the spherical morphology of the as-synthesized CdSe QDs. Figure (1) shows the particle size histograms of $0 \mathrm{~h}$ and $4 \mathrm{~h}$ samples where the average particle sizes were 4.5 and $9 \mathrm{~nm}$, respectively, confirming the success of this way to fabricate the CdSe QDs. Besides, this confirms the increase in size through the refluxing process as suggested by the XRD data.The d-spacing estimated from the lattice fringes (Fig. 2 a) was found to beabout $0.4 \mathrm{~nm}$ which is in excellent agreement with that of (111) plane of cubic CdSe as suggested by the XRD data mentioned above. Furthermore, the EDX spectra (Fig. 2 b) and the mapping spectra (Fig. 3) confirmed the existence of $\mathrm{Cd}$ and Se elements.

\section{XPS}

The X-ray photoelectron spectra (Fig. 2) wereemployed to further confirm the chemical states and compositions of the as-synthesized CdSe QDs; the XPS survey (Fig. 2 a) shows the presence of the $\mathrm{Cd}, \mathrm{Se}, \mathrm{O}, \mathrm{C}$ and Na elements. The HR-XPS of Cd 3d, Se 3d, and $\mathrm{O} 1 \mathrm{~s}$ are demonstrated illustrated in Figure $(2 \mathrm{~b}-\mathrm{d})$. Typically, the two sharp peaks at 404.5 $\mathrm{eV}$ and $411.2 \mathrm{eV}$ which are characteristic of $\mathrm{Cd} 3 \mathrm{~d}_{5 / 2}$ and $\mathrm{Cd} 3 \mathrm{~d}_{3 / 2}$, respectively, are observed (Fig. 2 b) ${ }^{[\mathbf{5 , 6}]}$. Moreover,the observed peaks at 53.21 and $54.15 \mathrm{eV}$ (Fig. 2 c) are corresponding to the binding energies of Se $3 \mathrm{~d}_{5 / 2}$ and Se $3 \mathrm{~d}_{3 / 2}$, respectively ${ }^{[5,6]}$.In addition to this, the HR-XPS of O 1s (Fig. 2 d) showed two peaks located at $531.19 \mathrm{eV}$ and $533.02 \mathrm{eV}$, which can be attributed to the $\mathrm{C}=\mathrm{O}$ and $\mathrm{C}-\mathrm{OH}$ species from the employed capping agents (TGA and TSC), while the higher O1s peak at $535.21 \mathrm{eV}$ represents the existence of adsorbed water $\left(\mathrm{H}_{2} \mathrm{O}\right)^{[25]}$. Furthermore, the absence of $\mathrm{SeO}$ peak located at $59 \mathrm{eV}$ in the obtained HR- 
Elhussein M. Hashem et al.

XPS indicates the superior stability of the as-synthesized samples in open-air conditions revealing the success of our facile synthesis route ${ }^{[19]}$.
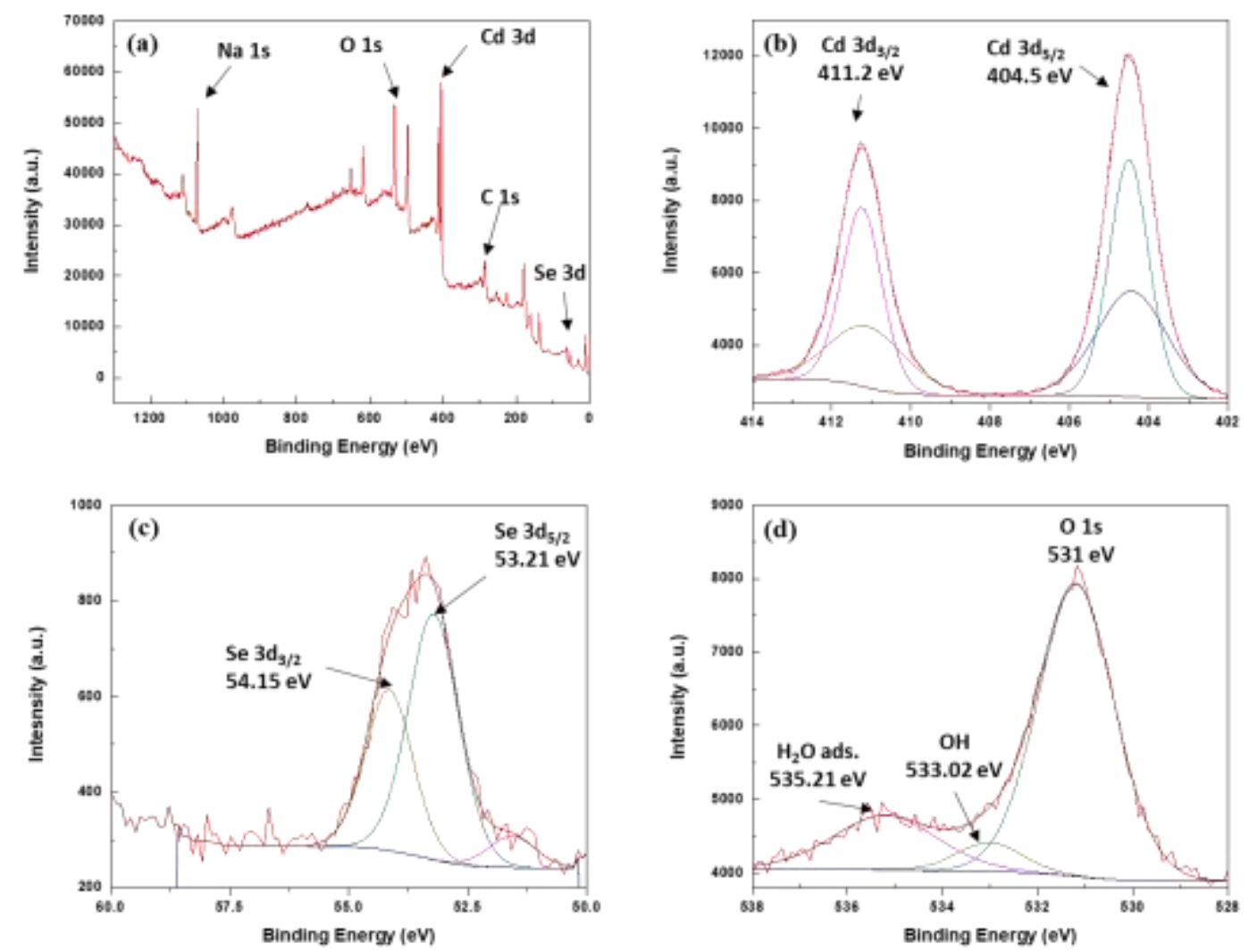

Fig. 2. The XPS survey (a), HR-XPS spectra of Cd 3d (b), Se 3d (c), and O 1s (d)

\subsection{Optical properties}

The UV-Visibleabsorption properties of semiconductors QDs play an important role in their photocatalytic applications. The UV-Visible absorption spectra (Fig. 3 a) showed that there is a gradual red shift with increasing the refluxing time, where the excitonic absorption peak varies from $\sim 444 \mathrm{~nm}$ to $560 \mathrm{~nm}$ at the end of the recrystallization process. This can be easily noticed throughthe visible change in color from yellow inthe case of $0 \mathrm{~h}$ sample to blood-red in 4h sample (Fig. 3 a: inset). This could be attributedto the increase in the particle size of the as-synthesized CdSe QDs, which is an excellent agreement with the XRD and HRTEM findings mentioned above.

Additionally, the recorded DRS measurements (Fig. 3 b) Conducted on CdSe QDs powders have revealed the red-shift in the absorption peaks that can be ascribed to the aggregation or growth of particles during the drying process. The bandgap energies of the assynthesized CdSe QDs were estimated to be 2.40, 2.20, 2.03, 2.00, $1.97 \mathrm{eV}$ for 0, 1, 2, 3, and $4 \mathrm{~h}$ samples, respectively, using Tauc plot (Fig. $3 \mathrm{c}$ ). It is essential to mention that the estimated bandgap energies of the fabricated CdSe QDs are higher than that of the bulk CdSe $(1.75 \mathrm{eV})$ which is attributed to the quantum size effect ${ }^{[19]}$.

Figure $(3 \mathrm{~d})$ showed that the PL peaks of the as-synthesized CdSe QDs ( $0 \mathrm{~h}$ and $1 \mathrm{~h}$ sample) exhibited very-broad bands with high intensities reflecting the defect-related emission, which also characterized by a red-shift within $1 \mathrm{~h}$ of refluxing due to the increase of particle size.This defect-related emission may be generated due to that the extremely smallnanocrystals have a very high surface-to-volume ratio leading to the formation of highdensityvacancies and trap sites on their surfaces are easy to be formed anddifficult to be 
entirely passivateddue to restriction created by the size ofthe capping agents' molecules ${ }^{[26]}$. On the contrary, unprecedented and unique blue shifts in the PL spectra in the case of $2 \mathrm{~h}$ and $3 \mathrm{~h}$. This may be explained by the disappearance of traps and defects due to the enhancement in crystallinity and morphology in samples within the refluxing process as the PL peaks become narrower and sharper. Moreover, the $4 \mathrm{~h}$ sample exhibited the typical redshiftbecause of the increase in size.

\section{Conclusion}

CdSe QDs of small size (4.5-9 $\mathrm{nm}$ ) have been successfullysynthesized through a facile one-pot approach. The prepared QDs exhibited excellent optical properties and unprecedented PL performance in the visible region. The refluxing time has a critical effect on both absorbance and bandgap energy as the absorbance varied from $440 \mathrm{~nm}$ to $560 \mathrm{~nm}$ and the bandgap energy from 2.40 to $1.97 \mathrm{eV}$.
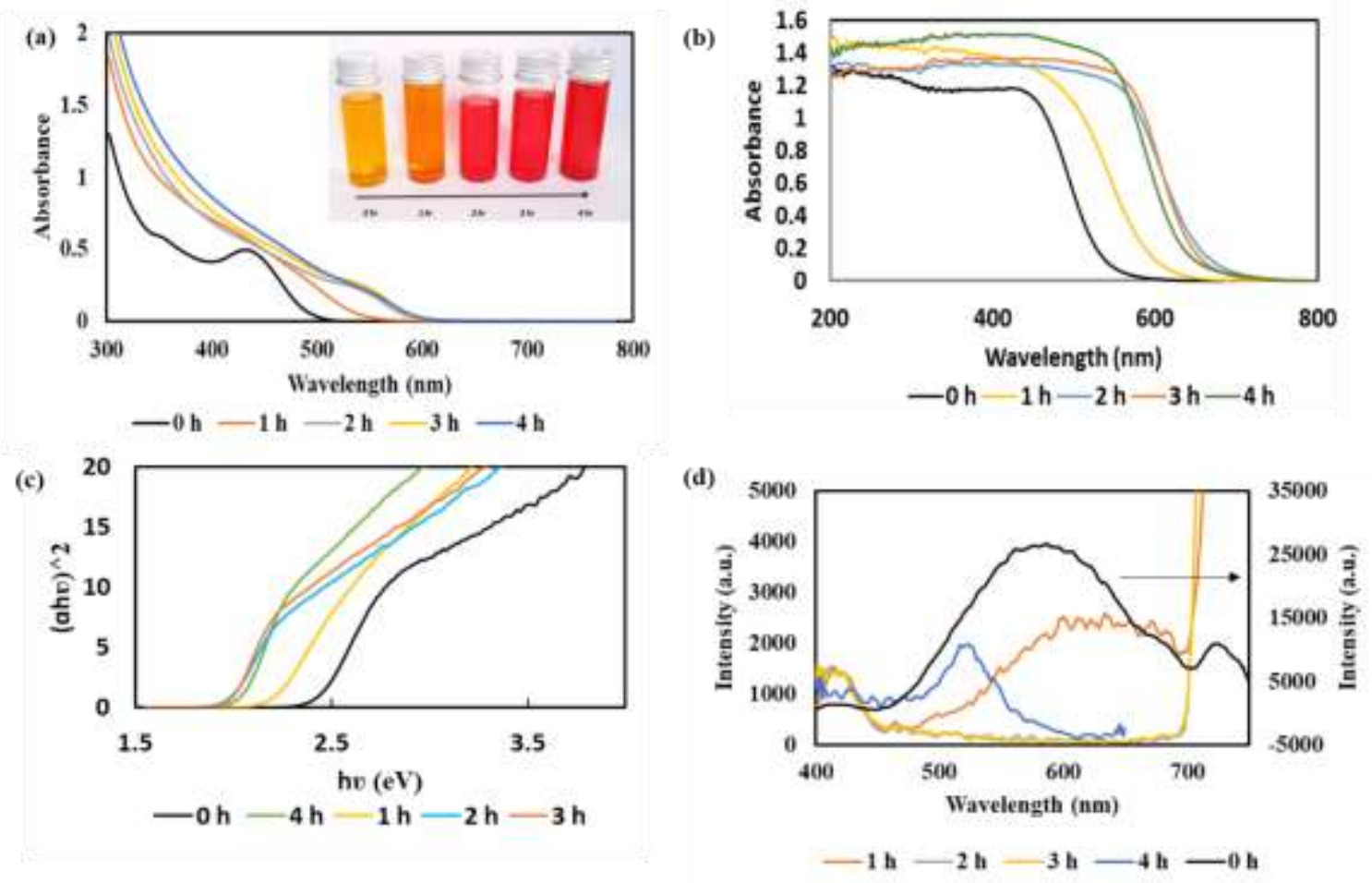

Fig. 3.The temporal evolution of UV-Visible absorbance (Solvent: water) (a), DRS (b) and Eg (Tauc plots) $(c)$, and the PL $\left(\lambda_{\text {excitation }}=370 \mathrm{~nm}\right)(\mathrm{d})$ of the as-synthesized CdSe QDs.

\section{REFERENCES}

1- M.A. Hamza; A.N. El-Shazly; S.A. Tolba and N.K. Allam (2020). Novel Bi-based photocatalysts with unprecedented visible light-driven hydrogen production rate: Experimental and DFT insights. Chem. Eng. J., 384 :123351. https://doi.org/10.1016/j.cej.2019.123351.

2- M.A. Hamza; A.N. El-Shazly and N.K. Allam (2020). Facile template-free one-pot roomtemperature synthesis of novel $\mathrm{m}-\mathrm{Bi}(\mathrm{OH}) \mathrm{CrO} 4$ microspheres. Mater. Lett. 262 : 127188. https://doi.org/10.1016/j.matlet.2019.127188.

3- H.L. Wu; X.B. Li; C.H. Tung and L.Z. Wu (2019). Semiconductor Quantum Dots: An Emerging Candidate for CO2 Photoreduction, Adv. Mater. 31 (2019) 19700709 (1-23). https://doi.org/10.1002/adma.201900709. 
Elhussein M. Hashem et al.

4- $\quad$ R. Gupta; W.J. Peveler; K. Lix and W.R. Algar (2019). Comparison of Semiconducting Polymer Dots and Semiconductor Quantum Dots for Smartphone-Based Fluorescence Assays. Anal. Chem. 91: 10955-10960. https://doi.org/10.1021/acs.analchem.9b02881.

5- $\quad$ D. Zhong; W. Liu; P. Tan; A. Zhu; Y. Liu; X. Xiong and J. Pan (2018). Insights into the synergy effect of anisotropic $\{001\}$ and $\{230\}$ facets of BaTiO3 nanocubes sensitized with CdSe quantum dots for photocatalytic water reduction. Appl. Catal. B Environ. 227:1-12. https://doi.org/10.1016/j.apcatb.2018.01.009.

6- $\quad$ P. Wang; X. Li; J. Fang; D. Li; J. Chen; X. Zhang; Y. Shao and Y. He (2016). A facile synthesis of CdSe quantum dots-decorated anatase $\mathrm{TiO} 2$ with exposed $\{001\}$ facets and its superior photocatalytic activity, Appl. Catal. B Environ. 181: 838-847. https://doi.org/10.1016/j.apcatb.2015.08.046.

7- C. Hunsur; Ravikumar, S. R and R.G. Balakrishna (2020). Surface modified glass substrate for sensing E. coli using highly stable and luminescent $\mathrm{CdSe} / \mathrm{CdS}$ core shell quantum dots, J. Photochem. Photobiol. B Biol. 204 : 111799. https://doi.org/10.1016/j.jphotobiol.2020.111799.

8- M. Yoshii; Y. Murata; Y. Nakabayashi; T. Ikeda; M. Fujishima and H. Tada (2016). Coverage control of $\mathrm{CdSe}$ quantum dots in the photodeposition on $\mathrm{TiO} 2$ for the photoelectrochemical solar hydrogen generation, J. Colloid Interface Sci. 474 :34-40. https://doi.org/10.1016/j.jcis.2016.04.018.

9- $\quad$ V.K. Gupta; A. Fakhri; M. Azad and S. Agarwal (2017). Synthesis of CdSe quantum dots decorated $\mathrm{SnO} 2$ nanotubes as anode for photo-assisted electrochemical degradation of hydrochlorothiazide: Kinetic process, J. Colloid Interface Sci. 508:575-582. https://doi.org/10.1016/j.jcis.2017.08.083.

10- T. Vanwie; E. Wysocki; J.R. Mcbride and S.J. Rosenthal (2019). Bright Cool White Emission from Ultrasmall CdSe Quantum Dots, Chem. Mater. 31:8558-8562. https://doi.org/10.1021/acs.chemmater.9b03718.

11- Z. Jin; A. Kapur; W. Wang; J.D. Hernandez; M. Thakur and H. Mattoussi (2019). The dual - function of lipoic acid groups as surface anchors and sulfhydryl reactive sites on polymer - stabilized QDs and Au nanocolloids. J. Chem. Phys., 151:164703. https://doi.org/10.1063/1.5126432.

12- N. Gheshlaghi; M. Faraji and H. Sedaghat (2019). Structural dependent, dielectric and conduction analysis of CdSe based quantum dots, SN Appl. Sci., 1:1-7. https://doi.org/10.1007/s42452-019-0451-2.

13- B. Samuel; S. Mathew; V.P.N. Nampoori and A. Mujeeb (2019). Defect passivation introduced through surface reconstruction in TOPO capped CdSe quantum dots for enhancement in quantum yield, Opt. Mater. (Amst)., 88: 204-209. https://doi.org/10.1016/j.optmat.2018.11.033.

14- T. Moriyasu; A. Kinan; Y. Baba and M. Kumakura (2020). Emission spectral change of CdSe / ZnS quantum dots caused by the dilution with organic solvents. J. Lumin., 117089. https://doi.org/10.1016/j.jlumin.2020.117089.

15- J. Augusto; F. Baum; E.C. Kohlrausch; F.C. Tavares; T. Pretto; F.P. Santos; J.F.L. Santos; S. Khan and M.J.L. (2019). Santos, 3-Mercaptopropionic, 4-Mercaptobenzoic, and Oleic Acid-Capped CdSe Quantum Dots: Interparticle Distance. Anchoring Groups, and Surface Passivation. J. Nanomater, 2019.

16- V. Ncapayi and O.S. Oluwafemi (2017). Green synthesis of anisotropic CdSe nanocrystals via a non -phosphine based method, Mater. Lett. 194 : 168-171. https://doi.org/10.1016/j.matlet.2017.02.039.

17- L.K. Putri; B.J. Ng; W.J. Ong; H.W. Lee; W.S. Chang; A.R. Mohamed and S.P. Chai (2020). Energy level tuning of CdSe colloidal quantum dots in ternary 0D-2D-2D CdSe QD/B-rGO/O-gC3N4 as photocatalysts for enhanced hydrogen generation, Appl. Catal. B 


\section{Facile nitrogen-free one-potaqueous synthesis of highly soluble and luminescent CdSe quantum dots}

Environ. 265: 118592. https://doi.org/10.1016/j.apcatb.2020.118592.

18- $\quad$ R. Mrad; M. Poggi; N. Ben Brahim; R. Ben Chaâbane and M. Negrerie (2019). Tailoring the photophysical properties and excitonic radiative decay of soluble CdSe quantum dots by controlling the ratio of capping thiol ligand. Materialia. 5 : 100191. https://doi.org/10.1016/j.mtla.2018.100191.

19- P. Wang; D. Li; J. Chen; X. Zhang; J. Xian; X. Yang; X. Zheng; X. Li and Y. Shao (2014). A novel and green method to synthesize CdSe quantum dots-modified $\mathrm{TiO} 2$ and its enhanced visible light photocatalytic activity, Appl. Catal. B Environ., 160-161: 217-226. https://doi.org/10.1016/j.apcatb.2014.05.032.

20- P.P. Ingole; R.M. Abhyankar; B.L.V. Prasad and S.K. Haram (2010). Citrate-capped quantum dots of CdSe for the selective photometric detection of silver ions in aqueous solutions, Mater. Sci. Eng. B Solid-State Mater. Adv. Technol. 168: 60-65. https://doi.org/10.1016/j.mseb.2009.10.033.

21- K. Surana; P.K. Singh; H.W. Rhee and B. Bhattacharya (2014). Synthesis, characterization and application of CdSe quantum dots. J. Ind. Eng. Chem., 20: 4188-4193. https://doi.org/10.1016/j.jiec.2014.01.019.

22- J. Wang and H. Han (2010). Hydrothermal synthesis of high-quality type-II CdTe/CdSe quantum dots with near-infrared fluorescence. J. Colloid Interface Sci., 351: 83-87. https://doi.org/10.1016/j.jcis.2010.07.025.

23- S. Zare and J. Tashkhourian (2020). Ultrasound-assisted synthesis of chiral cysteinecapped CdSe quantum dots for fluorometric differentiation and quantitation of tryptophan enantiomers. Microchim. Acta. 18 . https://doi.org/10.1007/s00604-019-4046-9.

24- M. Rana; A. Jain; V. Rani and P. Chowdhury(2020). Glutathione capped core/shell $\mathrm{CdSeS} / \mathrm{ZnS}$ quantum dots as a medical imaging tool for cancer cells. Inorg. Chem. Commun., 112: 107723. https://doi.org/10.1016/j.inoche.2019.107723.

25- D. Huang; J. Cui and X. Chen (2014). A morpholinium surfactant crystallization induced formation of Au nanoparticle sheet-like assemblies with uniform SERS activity, Colloids Surfaces A Physicochem. Eng. Asp. 456 : 100-107. https://doi.org/10.1016/j.colsurfa.2014.05.027.

26- Y.-S. Xia and C.-Q. (2008). Zhu, Aqueous synthesis of luminescent magic sized CdSe nanoclusters Yun-Sheng. Mater. Lett. 62:2103-2105. https://doi.org/10.1016/j.matlet.2007.11.027.

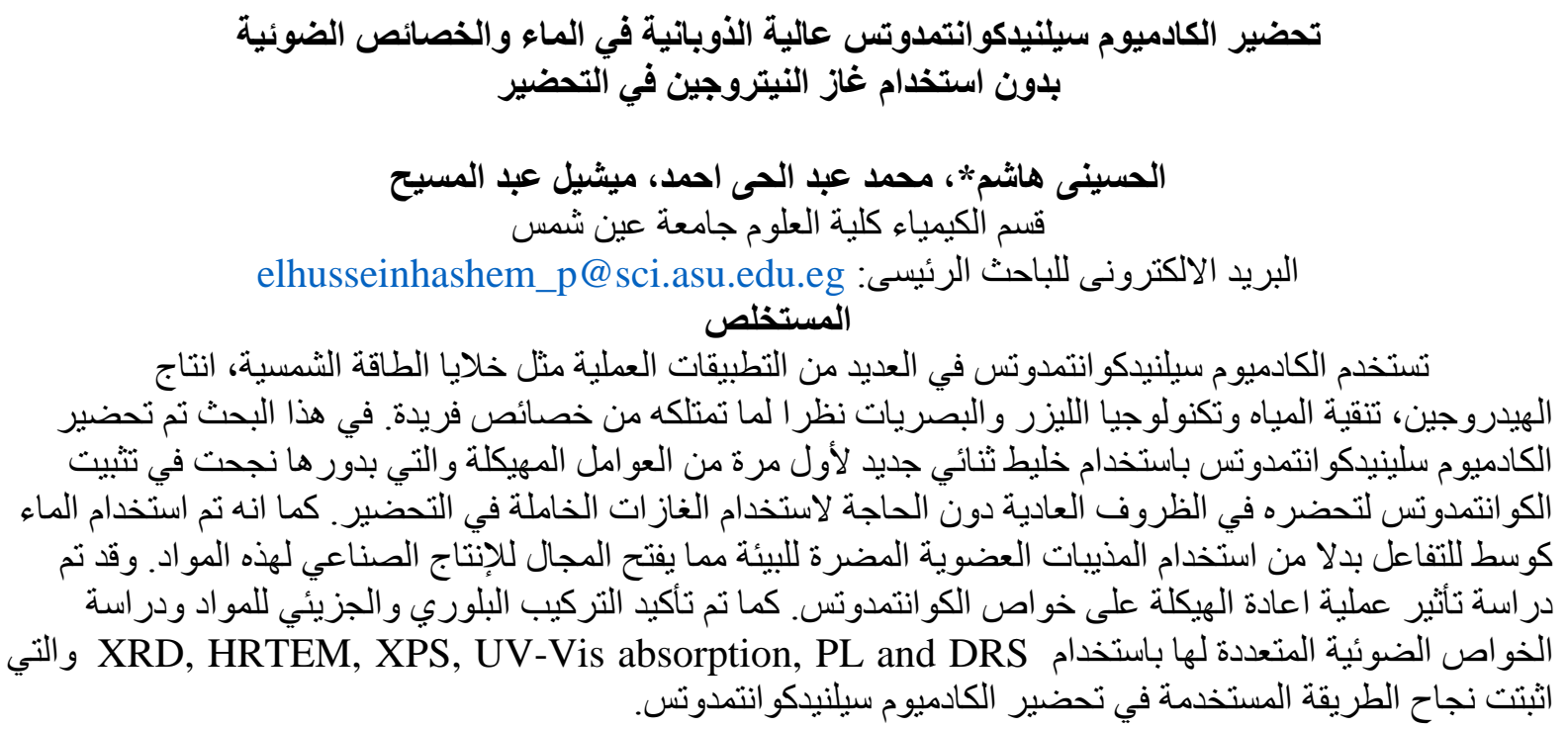

